\title{
BMJ Open Quality Implementation of a whole of hospital sepsis clinical pathway in a cancer hospital: impact on sepsis management, outcomes and costs
}

To cite: Thursky K, Lingaratnam S, Jayarajan J, et al. Implementation of a whole of hospital sepsis clinical pathway in a cancer hospital: impact on sepsis management, outcomes and costs.BMJ Open Quality 2018;7:e000355. doi:10.1136/ bmjoq-2018-000355

- Additional material is published online only. To view, please visit the journal online (http://dx.doi.org/10.1136/ bmjoq-2018-000355).

Received 9 February 2018 Revised 9 May 2018 Accepted 2 June 2018

Check for updates

For numbered affiliations see end of article.

Correspondence to Professor Karin Thursky; karin.thursky@mh.org.au

\section{ABSTRACT}

Infection and sepsis are common problems in cancer management affecting up to $45 \%$ of patients and are associated with significant morbidity, mortality and healthcare utilisation.

Objective To develop and implement a whole of hospital clinical pathway for the management of sepsis (SP) in a specialised cancer hospital and to measure the impact on patient outcomes and healthcare utilisation.

Methods A multidisciplinary sepsis working party was established. Process mapping of practices for recognition and management of sepsis was undertaken across all clinical areas. A clinical pathway document that supported nurse-initiated sepsis care, prompt antibiotic and fluid resuscitation was implemented. Process and outcome measures for patients with sepsis were collected preimplementation (April-December 2012), postimplementation cohorts (April-December 2013), and from January to December 2014.

Results 323 patients were evaluated (111 preimplementation, 212 postimplementation). More patients with sepsis had lactate measured $(75.0 \%$ vs $17.2 \%)$ and appropriate first dose antibiotic $(90.1 \%$ vs $76.1 \%$ ) (all $p<0.05$ ). Time to antibiotics was halved (55 vs $110 \mathrm{~min}, \mathrm{p}<0.05)$. Patients with sepsis had lower rates of intensive care unit admission (17.1\% vs $35.5 \%)$, postsepsis length of stay (7.5 vs 9.9 days), and sepsisrelated mortality $(5.0 \%$ vs $16.2 \%$ ) (all $p<0.05)$. Mean total hospital admission costs were lower in the SP cohort, with a significant difference in admission costs between historical and SP non-surgical groups of \$A8363 (95\% Cl 81.02 to $16645.32, p=0.048$ ) per patient on the pathway. A second cohort of 449 patients with sepsis from January to December 2014 demonstrated sustained improvement. Conclusions The SP was associated with significant improvement in patient outcomes and reduced costs. The SP has been sustained since 2013, and has been successfully implemented in another hospital with further implementations underway in Victoria.

\section{INTRODUCTION}

Infection and sepsis are common problems in cancer management affecting up to $45 \%$ of patients and are associated with significant morbidity, mortality and healthcare utilisation. $^{1-6}$ Patients with cancer are a unique population at increased risk of sepsis due to interventions such as chemotherapy, radiotherapy and complex or prolonged surgical procedures. However, most published international guidelines in this population focus on the management of neutropenic fever, and fail to address the full spectrum of patients across oncology, haematology, radiotherapy and cancer surgery, nor do they provide recommendations for the recognition and resuscitation of patients with sepsis. ${ }^{7-9}$ In a cancer hospital setting, sepsis is more common than neutropenic fever yet specialised guidelines are lacking. ${ }^{10}$

The major limitation of neutropenic fever guidelines is that the definition of neutropenic fever necessarily requires that the patient must have both fever and neutropaenia for initiation of antimicrobial therapy. ${ }^{7-9}$ In contrast, the diagnosis of sepsis using the systemic inflammatory response syndrome (SIRS) criteria or the more recent quick sepsis-related organ failure assessment (qSOFA) criteria, ${ }^{11}$ does not require the presence of fever. It is important to recognise that $30 \%$ of patients with severe sepsis do not have fever, and patients with neutropaenia may meet sepsis criteria without being febrile, leading to delays in administration of appropriate antimicrobial therapy. ${ }^{12}$

Standardisation of sepsis management using bundles of care has led to dramatic and sustained improvements in patients with severe sepsis or septic shock. ${ }^{13-17}$ We hypothesised that an optimal model for the management of infection in a cancer hospital would be to take a whole-of-systems approach to improving the recognition and resuscitation of sepsis in patients across their treatment journey, which might include surgery, chemotherapy and radiotherapy. 
We undertook a quality improvement project in our cancer centre to identify existing processes and barriers to effective and efficient management of sepsis across the hospital, and to implement a hospital-wide clinical sepsis pathway (SP). The hospital-wide sepsis initiative, including the SP was implemented in March 2013. The aims of this study were to examine the impact of the SP intervention on process measures for sepsis recognition and management, and outcomes such as intensive care unit (ICU) admission rates, hospital length of stay (LOS), mortality and hospitalisation costs.

\section{METHODS}

\section{Context}

Peter MacCallum Cancer Centre (PMCC) is a 100 inpatient-bed tertiary cancer hospital with haematology, medical oncology, cancer surgery and radiation oncology. As well as four wards, ambulatory areas include a chemotherapy day unit, a medical day unit, apheresis, hospitalin-the-home service, radiotherapy, diagnostic imaging and outpatient clinics. As there is no emergency department, patients were routinely admitted directly to the wards or through ambulatory areas. Cancer surgeries routinely performed include complex colorectal surgery (eg, pelvic exenteration), upper gastrointestinal surgery, breast and plastic surgery, head and neck surgery and sarcoma/skin cancer surgery. Neurosurgery, orthopaedic and thoracic surgery were generally performed at affiliated tertiary centres, although postoperative treatment including adjuvant radiotherapy and chemotherapy were administered at PMCC. Typically, patients present with sepsis at multiple sites throughout the hospital-medical day unit, ambulatory care/outpatients, chemotherapy day unit, apheresis, radiotherapy and inpatient wards. The hospital was moved to the Parkville precinct opposite Royal Melbourne Hospital in June 2016.

\section{Definitions}

The sepsis-2 (SIRS-based sepsis) criteria were used for this programme as it was developed prior to the publication of the sepsis-3 criteria. ${ }^{18}{ }^{19}$ Clinical review criteria were represented as Between the Flags on the ward observation chart: $\mathrm{SpO}_{2} 90 \%-95 \%$, heart rate (HR) 120-130 beats , respiratory rate (RR) 25-30 breaths per minute, systolic blood pressure (SBP) 90-100 $\mathrm{mm} \mathrm{Hg}$, temperature $>38^{\circ} \mathrm{C}$ or $<35.4^{\circ} \mathrm{C}$. Medical emergency team (MET) call criteria: $\mathrm{SpO}_{2}<90 \%, \mathrm{HR}>130$ beats per minute, $\mathrm{RR}$ $>30$ or $<6$ breaths per minute, $\mathrm{SBP}<90 \mathrm{~mm} \mathrm{Hg}$, altered conscious state, decreased urine output $<0.5 \mathrm{~mL} /$ hour for $>2$ hours. Appropriateness of antimicrobial therapy was based on the National Antimicrobial Prescribing survey definition, and is a composite measure of choice based on indication, clinical guidelines, dose, route and allergy label. ${ }^{20}$

\section{Overview of design}

A mixed methodology exploratory sequential design was used to investigate barriers associated with efficient and effective inpatient management of sepsis. ${ }^{21}$ Hospital administrative data including International Classification of Diseases (ICD)-10 coding, the hospital antimicrobial approvals system and MET calls were used define the scope, burden and cost of sepsis. Concurrently, focus group interviews using lean methodology were completed to provide a comprehensive overview of sepsis management across all areas of the hospital. Results were used to inform the development and implementation of an evidence-based SP with evaluation of process and outcome measures. Finally, the resulting hospitalisation costs as associated hospital LOS were estimated. The project commenced in December 2011.

\section{Focus group interviews}

A sepsis working party was formed with medical, nursing and pharmacy representatives from the antimicrobial stewardship team, infectious diseases department, inpatient wards, specialty units and outpatient/ambulatory units. Members were clinical champions in their own area during the programme. Each member undertook direct observation and focus group interviews in an allocated clinical area. Three key areas were examined: (i) issues relating the identification of sepsis; (ii) issues relating to clinical review of patients with sepsis and (iii) issues relating to timely administration of first dose of antibiotic.

The focus group interviews were conducted by members of the sepsis working party using purposive sampling of junior and senior medical and nursing staff working across the hospital during January and February 2012. Process mapping was used to identify educational, clinical and infrastructural barriers to the efficient and effective inpatient and outpatient management of sepsis. This subsequently guided the development and implementation of the SP as described in the 'Results' section.

\section{Impact analysis}

To estimate the burden of sepsis at PMCC, hospitalisations with an ICD-10AM (8th edition) coding for sepsis (online supplementary table 1$)^{22}$ were matched with hospital administrative data including ICU admission and 28-day mortality. Coding data were extracted from March 2011 to March 2014 inclusive. Rates of sepsis, ICU admission and 28-day mortality were presented per 1000 admissions.

The impact of the hospital-wide sepsis initiative was addressed through comparison of patient outcomes prepathway and postpathway implementation. For the prepathway (historical) cohort, patients were identified using hospital ICD-10AM coding and the antimicrobial approval system used for stewardship (Guidance, Melbourne Health, Australia). For the postpathway (SP) cohort, ICD-10AM coding and Guidance antimicrobial approval data were used in addition to the final audit page of each SP that was faxed to the Department of Infectious Diseases. Cases were also identified from the hospital-based electronic MET records system (RiskMan, Southbank, Victoria, Australia). Patients were excluded if 
they began treatment at another hospital or where sepsis onset was in the ICU. Demographic information (age, cancer diagnosis, admitting unit), sepsis recognition and management (vital signs, absolute neutrophil count, lactate level, fluid resuscitation and time-to-antibiotic) and outcome data (bacteraemia, ICU admission, hospital LOS, 30-day mortality) were collected from patient medical records.

The historical cohort presented from March to December 2012 and the SP cohort presented from March to December 2013, inclusive. A larger audit of all patients in the 2014 calendar year was retrospectively collected in 2015. Demographic information, sepsis recognition and management and outcome data in the historical and SP cohorts were compared and the SP cohort was further stratified by whether or not patients were entered onto the pathway. Administrative data and antimicrobial utilisation data were collected from 2011 to March 2016.

\section{Statistical and cost analysis}

A $\mathrm{X}^{2}$ test was used to compare categorical variables, and a Mann-Whitney U test was used to compare continuous non-parametric variables (Stata V.13.0, College Station, Texas, USA). All $p$ values were two-tailed, and $p<0.05$ was considered to be statistically significant.

Hospitalisation costs of patients from the historical and implementation cohorts were extracted from the hospital's administrative records, which reflected each patient's hospital resource consumption. Costs included both direct and indirect costs and were categorised into relevant resource-use groups (such as allied health, ICU, nursing, imaging, medical, pharmacy, surgical and outside hospital care) for the entire length of their admission. A subset analysis according to cancer treatment modality was conducted where all medical patients (including haematology, medical oncology and radiation oncology (HMR)) were compared with surgical oncology patients.

Total hospital admission cost was the sum of all resource-use groups. The resource-use specifications are listed in online supplementary table 2 . All mean costs of hospital resource use were reported with SD or $95 \%$ CIs. A t-test was used to test mean differences for those on the SP compared with the historical cohort and $\mathrm{p}$ values were reported. All costs were adjusted to 2017 Australian dollars using the Consumer Price Index. ${ }^{23}$

\section{RESULTS}

\section{Focus group interviews and gap analysis (February-March} 2012)

Ambulatory areas in the hospital were evaluated separately to the inpatient areas as there were differences in staffing, workflow and access to equipment for the treatment of sepsis. The process maps for both ambulatory and inpatient areas are shown in online supplementary figures 1 and 2, respectively. These findings were distilled into three key focus areas that identified major gaps and hence key areas for improvement (table 1).
1. Improve recognition and referral of sepsis across clinical areas.

2. Improve time to medical review.

3. Improved timeliness for the correct treatment of sepsis. The actions taken to address each area are described in table 1. Importantly, a new six-bed area for acutely unwell patients requiring higher acuity care was opened in December 2012 and was used to minimise direct admissions to the ward, and for unplanned admissions from ambulatory care areas.

\section{Sepsis pathway development (March-October 2012)}

Based on results from the process mapping, several strategies to optimise sepsis recognition and management were identified and implemented. Central to the initiative was the development and implementation of a hospital-wide clinical pathway for the initial management of sepsis (first 24 hours). The pathway was adapted from the Clinical Excellence Commission Adult Sepsis Kills pathway for emergency departments and focused on timely and appropriate recognition, response and referral of patients with sepsis. ${ }^{24}$ Notable modifications to the pathway included (i) adaptation for inpatient use, (ii) relevance to cancer and immunocompromised patients particularly with regard to antimicrobial choice and (iii) that the pathway would be nurse-initiated.

Introducing the SIRS criteria into an early warning definition for sepsis was a major practice change as criteria differed from the existing clinical review criteria and MET criteria. When and if a patient met the proposed pathway definition of sepsis (two or more SIRS criteria, or hypotension with SBP $<100 \mathrm{~mm} \mathrm{Hg}$ plus an infection), nursing staff would notify the doctor using the Identify, Situation, Background, Assessment and Recommendation criteria, ${ }^{25}$ and commence the pathway including intravenous cannulation, two sets of blood cultures, venous blood lactate and routine bloods. This addressed the major structural barrier to prompt treatment as intravenous cannulation is necessary to administer fluids and antibiotics. The doctor was then required to review the patient within $30 \mathrm{~min}$ to chart the antibiotics and fluid orders. If a patient fulfilled MET criteria, then this would trigger a medical emergency team response, or if the patient had Goals of Care criteria limiting care then this would be heeded. The initial fluid resuscitation volume $(10-20 \mathrm{mg} / \mathrm{kg})$ of crystalline fluid was to be given as a rapid fluid bolus if the patient was hypotensive or had an elevated lactate $(>4 \mathrm{mmol} / \mathrm{L})$. The empiric antimicrobial recommendations were chosen to cover major pathogens in neutropenic fever or intra-abdominal sepsis postcolorectal surgery (which were the highest risk groups in the hospital). The initial antibiotic choice was piperacillin/ tazobactam, cefepime for non-immediate hypersensitivity to beta-lactams, and ciprofloxacin plus vancomycin for immediate hypersensitivity to beta-lactams. Addition of gentamicin as a stat dose, and vancomycin were only recommended in the case of severe sepsis and in the case of vancomycin, additional indications for use such 
Table 1 Barriers to appropriate sepsis management and corresponding interventions

\section{Intervention}

\section{Issue focus 1: improve recognition and referral of sepsis across clinical areas}

\subsection{Target areas}

\section{Ambulatory areas:}

Staff rely on experience and training to identify sepsis; variability in referral practices, often have bank/junior staff.

Outpatient clinics: 300-400 visits per day, but 1 in 5 unplanned admissions due to sepsis. Strong support for a standardised approach.

Radiotherapy: perceived as infrequent: estimated as 1 every 2 months.

Apheresis: many other reasons for haemodynamic instability (eg, transfusion reactions).

\section{Inpatients areas:}

Different sepsis screening procedures across wards.

Many high-acuity patients (requiring emergency department level care) are admitted after-hours directly to wards.

Identification of sepsis without fever in patients is difficult; treatment guided by senior nurse/haematologists. Doctors often rationalise that fever is due to other causes (eg, cancer related).

\subsection{Observations}

Current chart uses clinical review (Between the Flags) parameters that differ from the systemic inflammatory response syndrome criteria. The basic observation charts in the ambulatory areas are not using the Between the Flags criteria. In radiotherapy, only a single set of observation done. No process to identify unwell patients.

Variation in compliance with RR measurement and documentation.

1.3 Knowledge gaps among junior resident medical offices (RMO) and nursing Whole of hospital education strategy involving staff about definitions, indicators and management of sepsis.

\section{Issue focus 2: improve time to medical review}

\subsection{Poor communication/handover:}

Doctors find it difficult to prioritise clinical review when vital clinical information is not available from the referring nurse.

Phones in wards/clinical areas left unattended or not answered.

Difficulty in troubleshooting with on-call doctors if not they are not familiar with complex haematology patients.

Many divisional junior staff do not have a formal morning handover process. Overnight events are usually picked up by reading clinical notes by the day teams.

\subsection{Absent or unclear escalation process}

Radiotherapy: notably more difficult to contact Radiation Oncology Registrars (as usually off-site for education).

Poor clarity about escalation process if multiple teams are involved (med oncology vs radiation oncology vs surgery).

Poor ownership of the medically unwell patient who is not a planned day admission; confusion about who to call (multiple teams). As above for inpatient areas.

\subsection{Medical emergency team (MET) calls}

Most MET calls $(60 \%)$ are after-hours when only skeleton junior medical staff are available.

No formal escalation process if RMO does not attend for clinical review in time. Nurses may revert to MET call if desperate.

Many inpatients that develop sepsis immediately postoperative have not been to a preadmission clinic; surgical RMOs unfamiliar and cases can be complex. Many high-acuity patients are admitted after-hours to the hospital.

\section{Issue focus 3: improve timeliness for correct treatment of sepsis}

3.1 Advanced care directives or not-for-resuscitation status

Not readily available for many patients (especially in ambulatory setting).
Sepsis pathway to be implemented across all ambulatory and inpatient areas.

Decision aids for sepsis identification and workup (posters) in all clinical areas.

six bed acute assessment area opened in December 2012. Used for all unintended admissions where possible. On same floor as the intensive care unit and able to monitor patients.

Observation charts standardised across all inpatient areas.

Observation chart adapted for single day to ambulatory areas including radiotherapy. Nursing education and audits of observations. junior, senior medical staff and nursing. Sepsis launch; posters and in-services
Institute Identify, Situation, Background, Assessment and Recommendation for nurses to page, communicate efficiently to doctors. Mobile phones provided to nurse unit managers and patient services.

ManagersInstitute formal handover process for overnight staff.

Institute handover between nursing shifts about patients with sepsis.

\section{Established an accepted escalation process}

Once implemented-wards to audit and feedback.
Change in RMO roster to that more staff onsite till 21:00 hours and to assist with late surgical admissions.

After-hours admissions sent to Acute Assessment Area ward. 


\subsection{Fluid resuscitation}

Not concordant with Australian/international guidelines-smaller volumes (than to $20 \mathrm{~mL} / \mathrm{kg}$ ) are commonly prescribed.

Fluid is not being administered as rapid fluid bolus (eg, increased maintenance fluids or using the intravenous pump on $999 \mathrm{~mL} / \mathrm{hour}$ ).

Basic fluid resuscitation does not commence until after medical review or MET called.

Many staff not familiar with the use of rapid infusers.

\subsection{Difficult intravenous cannulation and other equipment issues}

Lack of sufficient nurse intravenous cannulators-leading to major delays in instituting fluids/antibiotics (worse after-hours)

No intravenous trolley on haematology and radiation oncology ward (RMOs

have to get equipment from other sites), no resuscitation trolley in apheresis.

Standard disposable tourniquets are inadequate, especially for obese/difficult cannulations.

\subsection{Access to intravenous antibiotics in clinical areas}

Some areas already take phone orders for antibiotics.

Apheresis/radiotherapy: does not have on imprest antibiotics for penicillin-

allergic patients - can be a long wait from pharmacy.

No antibiotics currently on resuscitation trolley.

\section{Intervention}

Education of all clinical staff and ensured adequate stock ofrapid infusors.

Performance measures:

Time to commencement of fluid bolus.

\section{Addressed equipment shortages.}

Non-disposable tourniquets, Hartmann's placed on resuscitation trolley and checked daily. Urgent credentialing of new cannulators. Intravenous trolleys all wards.

Stocked all clinical areas with sepsis antibiotics. In selected high-risk patients pre-emptive charting of antibiotics permitted. as suspected line infection, or known colonisation or infection with methicillin-resistant Gram-positive bacteria (online supplementary figure 3 for the SP document).

The SP protocol underwent extensive review and endorsement by key stakeholders within the hospital including senior representatives from the medical oncology, haematology, surgical and infectious diseases clinical units, senior nurses and nursing education, intensive care, palliative care, ICU liaison staff and clinical pharmacists. The pathway was developed as a medical record document, which would be filed with the patient's record and would facilitate handover as well as high-quality clinical coding for sepsis. There were seven iterations of the pathway document prior to the first pilot.

\section{Cycle 1 (pilot)}

A small preimplementation pilot was performed in November and December 2012. The Medical Day Unit, haematology wards, the MET call team tested the form for usability and feasibility. The pathway was very well liked, and the only major change made to the document was the inclusion of the Goals of Care statement. This document was then printed as the medical record document and allocated a document identification number. During this period through to February 2013, education and training was commenced.

\section{Cycle 2 (implementation)}

The hospital-wide sepsis initiative, including the SP was implemented in March 2013. A comprehensive education campaign accompanied the SP implementation. A standardised presentation was developed and delivered to medical and nursing and pharmacy staff in all clinical areas by members of the sepsis-working group. Weekly email communiqués summarising key management principles and real-time audit results were also distributed to all clinical staff. A series of multichoice questions emailed to all staff was a popular initiative (online supplementary figure 2). Time to antibiotic initiation was the process measure that nursing staff preferred as best measure of performance in each clinical area. They became key drivers of the programme across the hospital.

\section{Impact analysis}

Overall, 323 patients were included in preimplementation and postimplementation evaluation. There were 111 patients in the historical cohort and 212 patients in the postimplementation cohort with 176 patients on the SP and 36 patients not on SP. Details of demographics, process measures and key outcomes are provided in table 2 .

Both cohorts were not significantly different except for the higher proportion of surgical oncology patients $(22.7 \%$ vs $11.7 \%)$ and patients having surgery within 30 days $(18.3 \%$ vs $6.3 \%)$ in the SP cohort (all $\mathrm{p}<0.05)$. The majority of surgical patients on the SP $(85.0 \%)$ underwent colorectal $(62.5 \%)$ and gastrointestinal surgery $(22.5 \%)$.

Following introduction of the SP, a significantly higher proportion of patients had lactate measured $(75.0 \%$ vs $17.2 \%)$ and received the appropriate first antibiotic $(90.1 \%$ vs $76.1 \%) \quad($ all $\mathrm{p}<0.05)$ while time to first dose of antibiotics was significantly shorter (55 vs $110 \mathrm{~min}, \mathrm{p}<0.05$ ) in patients on the SP compared with the historical patient cohort. The defined daily doses (DDD)/1000 occupied bed-days of vancomycin and meropenem also fell after the pathway was 
Table 2 Patient demographics and clinical impact of implementation of whole of hospital sepsis pathway

\begin{tabular}{|c|c|c|c|c|c|}
\hline & $\begin{array}{l}\text { Historical cohort } \\
\text { (April-December } \\
\text { 2012) }\end{array}$ & \multicolumn{2}{|c|}{$\begin{array}{l}\text { Post-implementation cohort } \\
\text { (April-December 2013) }\end{array}$} & \multicolumn{2}{|c|}{$\begin{array}{l}\text { Year } 2 \text { of pathway } \\
\text { (January-December 2014) }\end{array}$} \\
\hline Admitted patient group, $\mathrm{n}(\%)$ & $111(100 \%)$ & $176(100 \%)$ & $36(100 \%)$ & $322(100 \%)$ & $127(100 \%)$ \\
\hline Medical oncology, $\mathrm{n}(\%)$ & $27(24.2)$ & $40(22.7)$ & $8(22.2)$ & 75 (23.3) & $37(29.1)$ \\
\hline Radiation oncology, n (\%) & $14(12.6)$ & $9(5.1)$ & $7(19.4)$ & $32(9.9)$ & $13(10.2)$ \\
\hline Male, n (\%) & $67(60.4)$ & $101(57.4)$ & $23(63.9)$ & $184(54.1 \%)$ & 85 (66.9\%) \\
\hline Hypotensive $<100$ mm Hg, n (\%) & $19(17)$ & $31(18)$ & $7(19)$ & $28(8.7)$ & $11(8.7)$ \\
\hline Heart rate, mean (SD) & $118(21.2)$ & $120(22.9)$ & $108(19.2)$ & $103(18.1)$ & $101(19.7)$ \\
\hline Respiratory rate, mean (SD) & $22.6(19.3)$ & $21.1(4.6)$ & $21.6(4.7)$ & $19.9(5.9)$ & $20.5(4.5)$ \\
\hline Surgery last 30 days, $\mathrm{n}(\%)$ & $7(6.3)$ & $32(18.3)^{\star}$ & $6(16.7)$ & $51(15.9)$ & $42(33.1)$ \\
\hline Two sets of BC prior to antibiotics, $\mathrm{n}(\%)$ & $56(50.4)$ & $131(74.4) \dagger$ & $23(63.9)$ & $200(62.1) \dagger$ & $26(28.4)$ \\
\hline Lactate level done, $\mathrm{n}(\%)$ & $19(17.2)$ & $132(75.0)^{\star}$ & $21(58.3)$ & $243(75.5)$ & $56(44.1)$ \\
\hline Lactate level $\geq 4, n$ (\%) & 2/19 (10.5) & 3/132 (2.2) & $3 / 21(14.2)$ & $7 / 243(2.9)$ & $5 / 56(8.9)$ \\
\hline Rapid fluid bolus if $\mathrm{SBP}<100 \mathrm{~mm} \mathrm{Hg}$ & $12 / 19(63.2)$ & $22 / 31(70.9)$ & $5 / 7(71.4)$ & $11 / 28(39.3)$ & 2/11 (18.2) \\
\hline Mean fluid bolus volume $(\mathrm{mL})$ & 423 & 512 & 542 & 642 & 400 \\
\hline Median time to antibiotics, min (range) & $110(0-3010)$ & $55(0-660)^{\star}$ & $60(0-405)$ & $60.5(5-1400)$ & $175(10-1245)$ \\
\hline Appropriateł first antibiotic, $\mathrm{n}(\%)$ & $85(76.1)$ & $156(88.4)^{*} / \dagger$ & $27(75.0)$ & $306(95)$ & $96(75.6)$ \\
\hline Bacteraemia, n (\%) & $56(50.5)$ & $52(29.1)$ & $16(44.4)$ & 63 (19.6) & $21(16.5)$ \\
\hline Appropriate initial antibiotic for $\mathrm{BC}$ & $45 / 56(80.4)$ & $47 / 52(90.4)$ & $14 / 16(87.5)$ & $60 / 63(95.3)$ & $18 / 21(85.7)$ \\
\hline 30-Day all-cause mortality, n (\%) & $21(18.9)$ & $13(7.4)^{\star}$ & $6(16.7)$ & $26(8.1)$ & $18(14.2)$ \\
\hline
\end{tabular}

${ }^{*} \mathrm{P}<0.05$ comparing historical and pathway cohort.

$\dagger \mathrm{P}<0.05$ comparing pathway cohorts from postimplementation and year 2 .

$\ddagger$ There were only three cases with a multidrug-resistant Gram-negative pathogen and three cases with multidrug-resistant Gram-positive pathogens for which the initial antibiotic therapy did not cover the pathogen.

BC, blood culture; ICU, intensive care unit; LOS, length of stay; SBP, systolic blood pressure.

implemented (figure 1). Medical oncology patients on the SP received antibiotics most rapidly (45 min, IQR 30-100 min) followed by haematology patients (60 min, IQR 30-100 min), radiation oncology (60 min, IQR 38-272 $\mathrm{min}$ ) and surgical patients (90 min, IQR 52-163 min), respectively.

Patients in the SP cohort on the pathway had significantly lower rates of ICU admission $(17.1 \%$ vs $35.5 \%)$ and ICU LOS (7.5 vs 9.9 days) (all $\mathrm{p}<0.05)$. Sepsis-related mortality $(5.0 \%$ vs $16.2 \%, \mathrm{p}<0.05)$ and 30 -day all-cause mortality $(7.4 \%$ vs $18.9 \%, \mathrm{p}<0.05)$ were also significantly lower following SP implementation.

The proportion of patients with normal SBP at sepsis recognition was lower in year 2 suggesting earlier recognition, although similar proportion required ICU admission (17.1\% vs $18.3 \%$ in year 1 and year 2$)$. There was poor compliance with the use of rapid bolus as well as recommended volumes. This may have been due to the fluid calculation required (by $\mathrm{mL} / \mathrm{kg}$ ) and general reluctance by medical staff to give larger boluses. The compliance with remainder of the pathway elements was maintained in the year 2 cohort from 2014 with improved antimicrobial appropriateness, but a reduction in the proportion of patients with two set of blood cultures. The pathway was associated with similarly improved patient outcomes. In year 2, there were a higher proportion of surgical patients and solid tumour patients in the non-pathway group.

The administrative coding data demonstrated that there was increased ascertainment of sepsis cases after the 


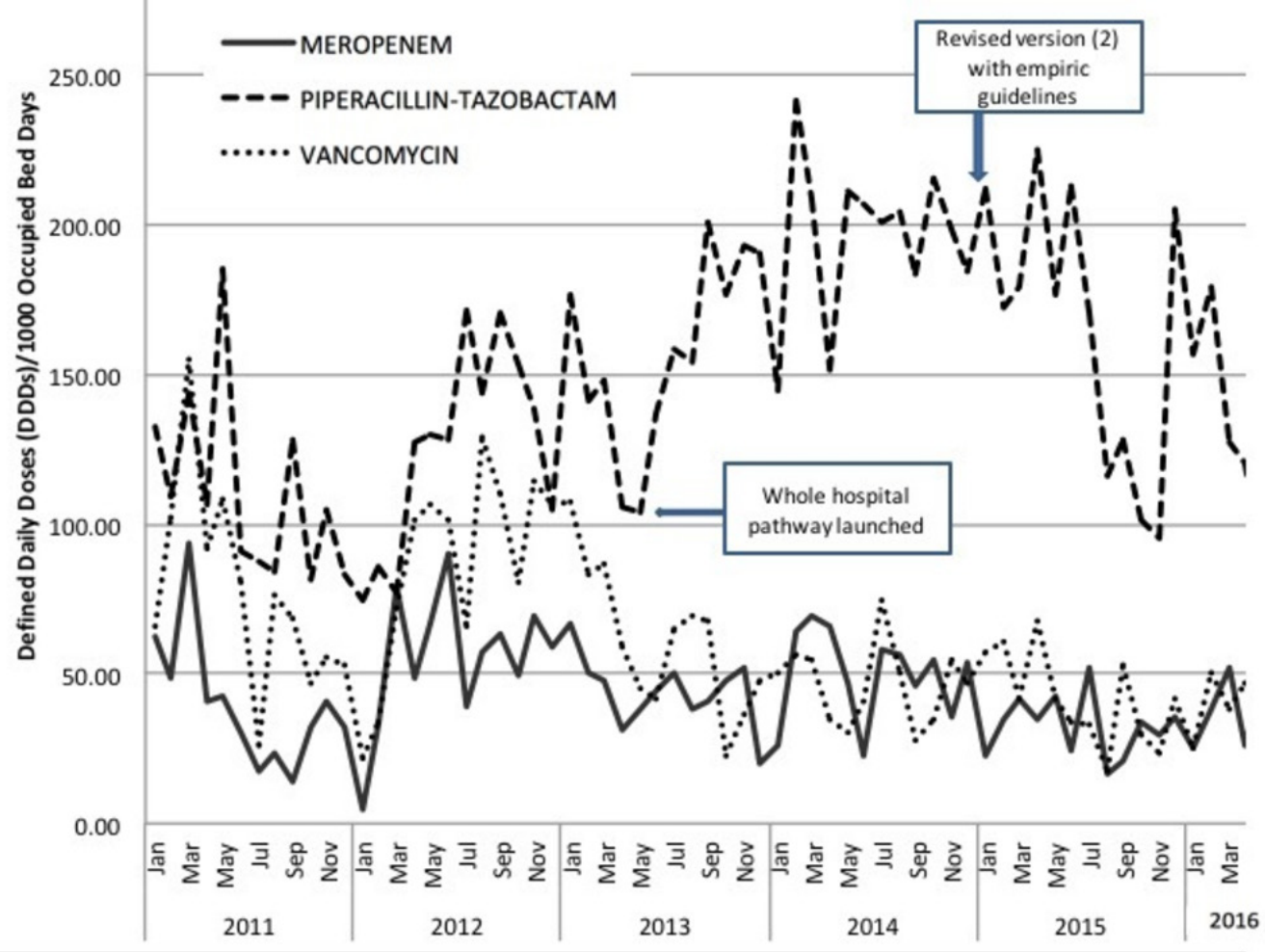

Figure 1 Impact of the sepsis pathway on utilisation of piperacillin/tazobactam, meropenem and vancomycin (as defined daily doses (DDDs)/1000 occupied bed-days.

implementation but no concomitant increase in all-cause mortality or increased ICU admission (figure 2).

\section{Cost analysis of implementation}

Full costing data were available for 275 patients (91 historical and $184 \mathrm{SP}$ cohorts). In the historical cohort, there were 80 HMR patients $(87.9 \%)$ and 11 surgical patients $(12.1 \%)$ while there were $147 \mathrm{HMR}$ patients $(79.8 \%)$ and 37 surgical patients $(20.1 \%)$ in the SP cohort.

Table 3 shows the mean total hospitalisation cost per patient across the two cohorts and for each of the patient groups. Surgical patients incurred higher total admission costs compared with the HMR patient group. For

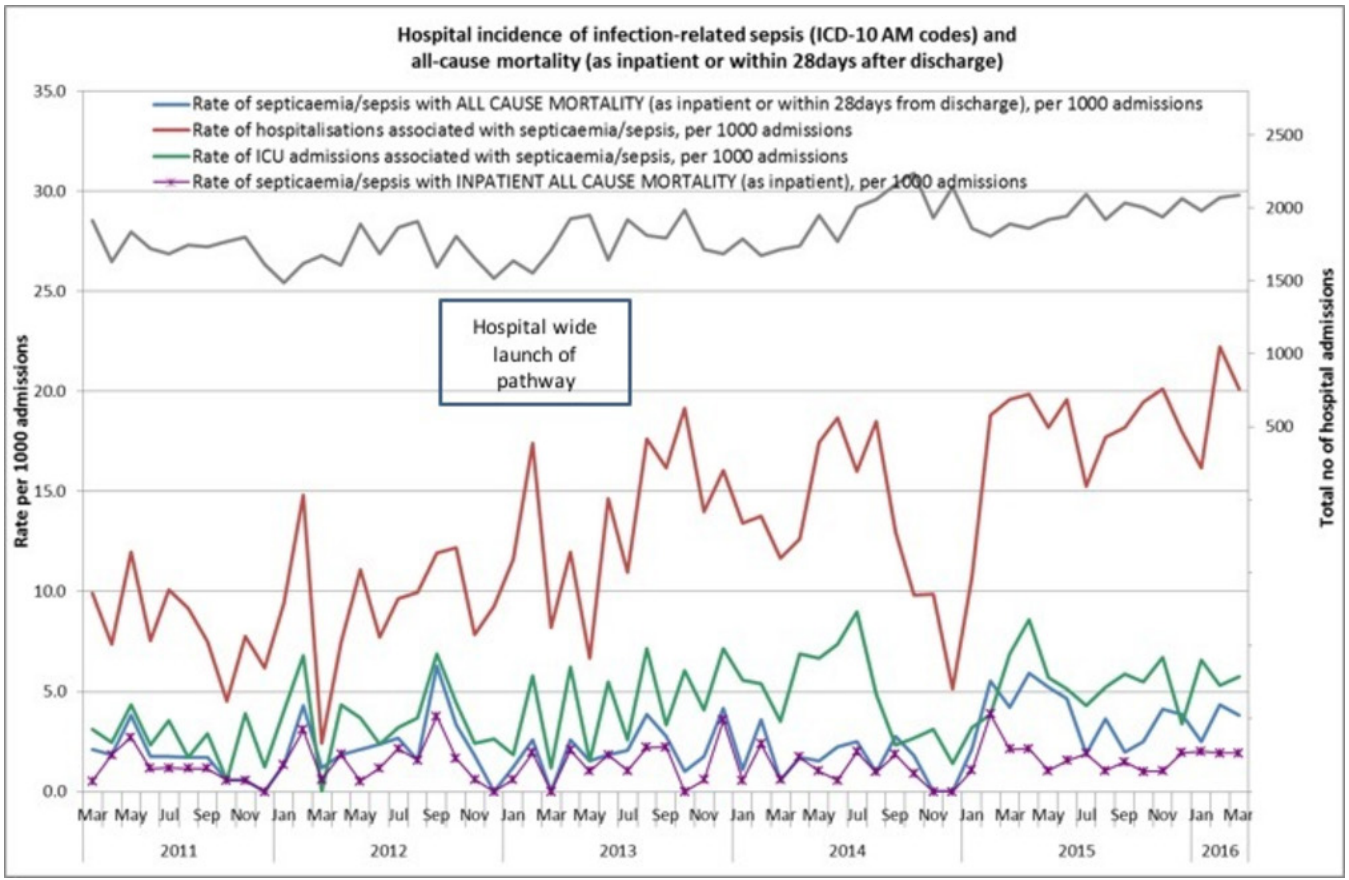

Figure 2 Burden of sepsis estimated from administrative data using ICD-10AM code preimplementation and postimplementation of sepsis pathway. ICD-10, International Classification of Diseases (ICD)-10; ICU, intensive care unit. 
Table 3 Overall mean total hospitalisation cost (2017 Australian dollars) for historical and sepsis pathway cohorts by patient groups

\begin{tabular}{llrrrrr}
\hline Patient group & Cohort & N & Mean total cost (\$A) & & 95\% CI & Mean difference (\$A) \\
\hline HMR & Historical & 80 & 41416.51 & 33603.44 & 49229.58 & \\
& SP & 147 & 33053.33 & 28609.06 & 37497.61 & $8363.17^{*}$ \\
Surgical & Historical & 11 & 102446.10 & -273.96 & 205166.1 & \\
& SP & 37 & 62334.73 & 33518.61 & 91150.86 & 40111.32 \\
\hline
\end{tabular}

${ }^{*} \mathrm{P}<0.05$.

HMR, haematology, medical oncology and radiation oncology; SP, sepsis pathway.

both groups, the mean total admission cost was lower in the SP cohort. There was a significant difference in the admission cost per patient on the pathway between the historical and SP HMR groups of \$A8363.17 (95\% CI $\$ \mathrm{~A} 81.02$ to $\$ \mathrm{~A} 16$ 645.32, $\mathrm{p}=0.048)$. Total admission cost was reflective of the hospital LOS.

For the majority of the resource-use groups (allied health, ICU, nursing, imaging, medical, pharmacy, surgical and outside hospital care), the cost incurred by historical cohort patients was higher than those in the SP cohort. The only resource-use group in which the SP cohort showed a higher cost than the historical cohort in both HMR and surgical groups was for pathology. There was a significant difference in ICU costs between the historical and SP cohorts of $\$$ A5470 (95\% CI \$A1780.219161.79, $\mathrm{p}<0.01$ ) for HMR patients. For patients in the HMR group, the bulk of the total admission cost (between $36 \%$ and $41 \%$ ) were for nursing services while in surgical patients, the majority of the cost was attributed to ICU cost $(29 \%$ and $30 \%)$.

\section{Cycle 3 (postimplementation and sustainability) 2014-2015}

The Sepsis Working Party continued to monitor the programme with regular feedback from nurse unit managers in each area. While education relating to the pathway was integrated into medical staff orientation and nursing in-services, aspects of the pathway for which further education was required included: rapid volume fluid resuscitation and universal lactate testing (even if the SBP was normal). The pathway was modified at the end of a detailed audit of patients in 2014 with modification of fluids based on weight, lowering the lactate cut-off, clearer indications for antibiotics and inclusion of an empiric antibiotic table to assist definitive therapy, prompts for review of microbiology and clearer instructions for monitoring (see table 2 and supplement for version 2 SP document, MR63 SEPSIS Pathway_2015). This was associated with maintained low usage of meropenem and vancomycin, and a reduction in piperacillin/ tazobactam (figure 1).

\section{Sustainability and expansion of the SP pathway to other sites (2015-2018)}

In June 2015, version 2 of the pathway was successfully implemented as part of the Western and Central Integrated Cancer Services programme into the oncology/ haematology and bone marrow transplant unit at the Royal Melbourne Hospital (RMH). In 2016, the RMH (led by KT) was funded by Better Care Victoria (BCV) to implement the SP across the whole of the hospital and was formally launched in 2017 as the 'Think Sepsis, Act Fast' programme. The modification of the SP, implementation and results of this project will be published elsewhere, but this version is now adopted as the current version in PMCC (see online supplementary file) and retains the $30 \mathrm{~min}$ time to antibiotics recommendation for cancer patients. The key modifications of the pathway are included in table 4. In October 2017, BCV funded the scale up of the Think Sepsis, Act Fast programme to 23 hospitalsand 11 health services in Victoria.

\section{DISCUSSION}

The introduction of a hospital-wide clinical pathway for the management of sepsis in a cancer hospital was associated with significant improvement in compliance with the core elements of the pathway and led to improved patient outcomes and reduced hospitalisation costs. This is the first study to examine the costs of sepsis in both neutropenic and non-neutropenic patients with cancer including surgical sepsis. ${ }^{26-29}$ The pathway was formally adopted into the partnering tertiary teaching hospital (Royal Melbourne Hospital) and has been endorsed by the Victorian state government for implementation into 26 further hospitals.

Process mapping the care of patients with sepsis, presenting key issues visually and as a gap analysis were essential to identify the core elements of the clinical pathway, to introduce structural changes around antimicrobials, venous blood lactate and fluid management and to identify the optimal mode of education by healthcare workers. Implementation studies of sepsis protocols have highlighted the importance of identifying structural barriers, knowledge gaps and competing priorities that exist in the hospital environment. ${ }^{30} 31 \mathrm{~A}$ recognition that patients with cancer presented with sepsis in multiple and diverse environments in the hospital such as chemotherapy day unit, surgery ${ }^{32}$ outpatients, or even radiotherapy, staffed by clinical staff with different skill levels, highlighted the need for a standardised and broad reaching approach. Different observation charts were used across areas and triggers for clinical review and 


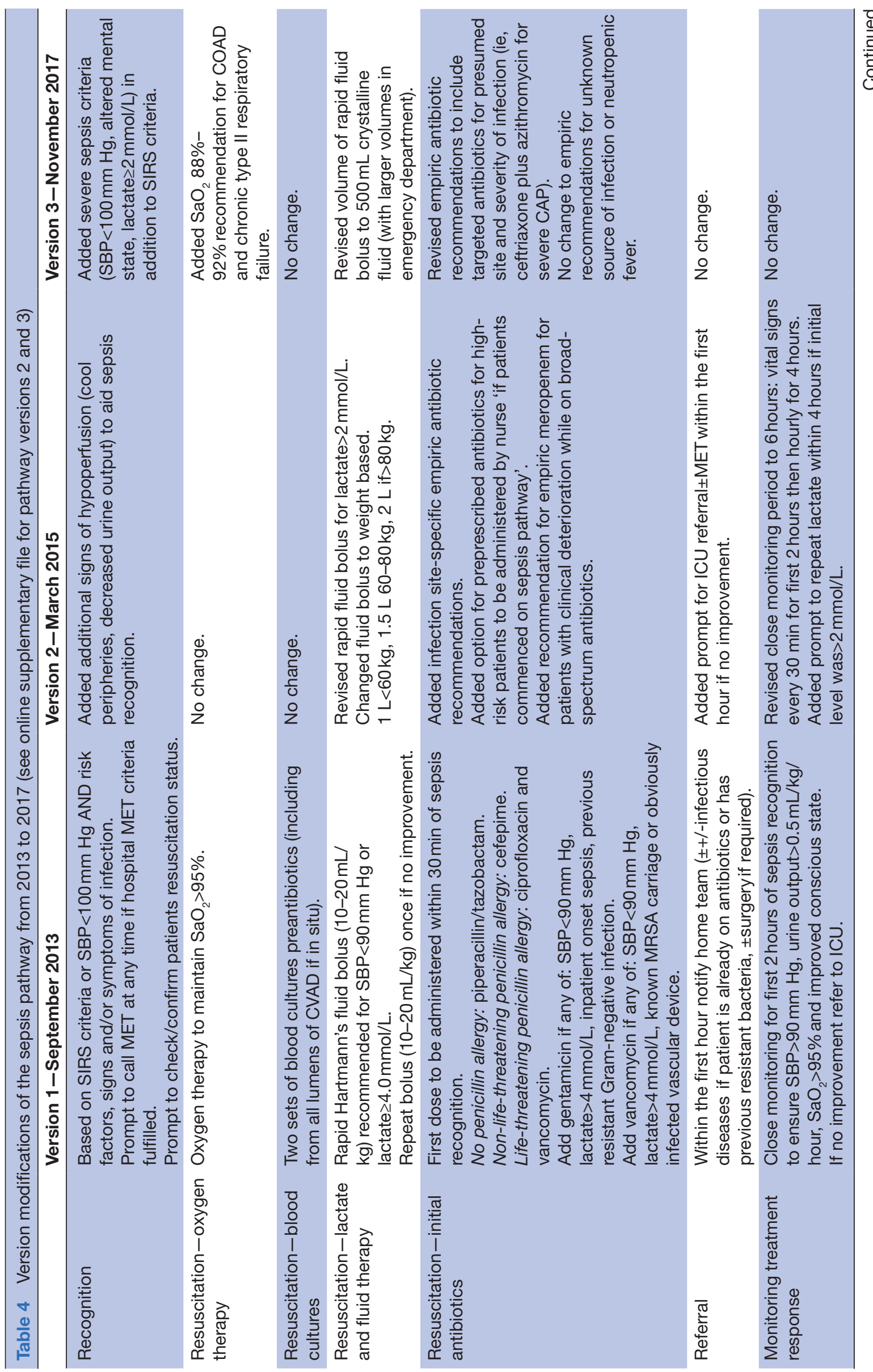


MET were not as sensitive as the SIRS criteria. Despite the potential for confusion, nursing staff embraced the new definition. Another important structural barrier identified was the lack of nursing staff able to cannulate, leading to delays in initiation of sepsis treatment while waiting for busy junior medical staff. Making available antibiotics for sepsis, and adequate numbers of rapid infusers for fluid administration on each ward was also necessary, as well as standardising the fluid on resuscitation trolleys.

In the cancer setting, nursing staff are accustomed to pathways and protocols for administration of analgesia and chemotherapy. In some clinical areas (eg, haematology), nursing expertise in managing very unwell patients led to a rapid adoption of the pathway. In other areas (eg, radiotherapy inpatients and surgery), more nurses needed more education and credentialing for procedures. A key facilitator for the success of this pathway was the change in practice to permit nurse-initiated care once a patient met sepsis criteria. Nurse-initiated care in sepsis has been successful in the emergency department and ICU setting. ${ }^{33} 34$ It also highlights the importance of clinical practices that facilitate staff empowerment and cultural changes, well-illustrated in mixed methods study of an implementation of a Rapid Response System in Northern Ireland.$^{35}$ Senior medical staff support is another critical factor that has been shown to impact on efforts to change public hospital systems. ${ }^{36}$

While there is a perception that patients with cancer are somehow different, and that neutropenic fever is the most common manifestation of infection, the preimplementation and postimplementation data confirmed that only $40 \%$ of patients were neutropenic at the time of sepsis. Neutropenic fever largely influences empiric antibiotic choice. The Australian national consensus guidelines for the empiric management of neutropenic fever ${ }^{7}$ formed the basis of the initial antimicrobial recommendations. Piperacillin-tazobactam was also considered an appropriate option for the treatment of common infections (intra-abdominal sepsis, healthcare-associated pneumonia) acknowledging that the pathway managed the first 24 hours and that antibiotics would be reviewed following the sepsis workup. Appropriateness of antimicrobial prescribing increased from $75 \%$ to $90 \%$, and was associated with a reduction in use of vancomycin and carbapenems, presumably as these were not recommended as part of the initial therapy. The overall usage of piperacillin-tazobactam (by DDDs) did increase, and this was addressed by including more directed options in the subsequent version of the SP.

Auditing of fluid administration from the paper medical records was difficult in the absence of an electronic medical record (EMR). The bolus fluid volumes, while increased, usually did not meet the $10-20 / \mathrm{kg}$ recommendation. There was an overall reluctance by medical staff to chart initial volumes $>500 \mathrm{~mL}$ and subsequent iterations of the pathway have led to this volume as the preferred initial bolus (see version 3 (MR63_SP_2017), supplement). 
With the publication of the updated sepsis criteria in 2016 and the adoption of the pathway by the RMH in 2017, the decision was made to retain the SIRS-based sepsis criteria as an early warning score but to include elements of severe sepsis (lactate, hypotension and altered mental state) (see version 3 MR63_SP_2017 , supplement). The results of the RMH implementation and the performance of pathway will be published separately. More recent publications including systematic reviews have demonstrated that the SIRS-based criteria correctly identifies one-third more patients with sepsis than does the qSOFA and may over diagnose sepsis, ${ }^{37} 38$ whereas qSOFA is slightly more specific and effective in predicting mortality from sepsis. In another study outside of ICU, patient with sepsis met $\geq 2$ SIRS criteria 12 hours earlier than $\geq 2$ qSOFA criteria. ${ }^{39}$ We believe that clinical pathways for high-risk patients should support the use of early warning criteria.

This hospital did not have an EMR where an alert trigger could be implemented using clinical observational criteria. The current evidence suggests that these alerts may be an effective intervention for sepsis. ${ }^{40-42}$ Nevertheless, this study demonstrates that the use of a paper-based clinical pathway using the SIRS-based sepsis definition can significantly improve the early appropriate treatment for sepsis. The clinical pathway was introduced as a formal medical record document that remained in the patient history and reinforced the programme's importance to the clinicians and nurses. In future, the pathway could be adapted to an EMR order set format. The use of the document also enhanced the quality of the sepsis auditing process and medical coding (ICD-10AM), which we identified in the preimplementation period as being poorly done, and an observation that has been already reported. ${ }^{434}$

This is the first study to examine the costs of sepsis in both medical and surgical patients with cancer. The implementation of the SP protocol led to a significant reduction in patient hospitalisation costs and importantly, a significant improvement in 30-day all-cause mortality. While this study further adds to existing literature linking the effectiveness of protocols or sepsis bundles that better manage sepsis in reducing patient mortality, ${ }^{13-1745}$ there is limited literature describing the economic impact of implementing a hospital-wide sepsis management pathway in patients with cancer. The implementation of SP in this study resulted in at least an average $\$$ A8363 reduction in hospitalisation cost per patient on the pathway compared with patients in the historical control group, which was largely driven by the shorter LOS. The lack of statistically significant difference in the surgical group was likely to be due to small patient numbers and large CIs. This reduction was translated across all resource-use groups except for pathology. Costing data were based on hospital cost rather than hospital charges, hence provide a true reflection of the healthcare resource used. These results highlight the SP protocol's potential to improve the hospital's efficiency in managing sepsis without compromising patients' safety and outcomes and can similarly be used to assess and inform the allocation of resources to ensure sustainability.

While this study demonstrated that implementation of a hospital-wide SP in a cancer centre is feasible, may improve key patient outcomes and also potentially reduce hospitalisation costs, undertaking the evaluation study had some limitations. A retrospectively identified historical cohort preimplementation was used as the comparator to the postimplementation SP cohort. The quality of medical coding for sepsis significantly improved after the implementation of the pathway (from $63.7 \%$ to $81.9 \%$ ), and was reflected in increased ascertainment of patients, and in particular the surgical cohort. We used additional data to accurately identify patients with sepsis. Use of administrative data for sepsis epidemiology and costing warrants further investigation. ${ }^{44}$ The compliance in process measures in patients who were not placed on the pathway was similar to those patients on the pathway, demonstrating that widespread practice change occurred after implementation. As this study was not a clinical trial, we cannot completely rule out other factors such as change in practice that might contribute to the difference in outcomes and costs that we observed. While the impact of the pathway was sustained, a more detailed understanding of why patients were not put on the pathway is necessary. Potential reasons include postsurgical patients who were not considered to be septic, end-stage cancer but without goals of care documentation or an oversight by busy clinical staff.

There is now compelling evidence that clinical pathways and bundles for the recognition and management of sepsis should be standard of care across hospitals. However, the implementation of a pathway requires a careful approach to understand the structural barriers and often unique issues experienced by clinical staff in both ambulatory and inpatient hospital areas. Antimicrobial stewardship programmes are ideally placed to lead a hospital-wide programme and to generate buy-in across clinical areas. ${ }^{47}$

\section{Author affiliations}

${ }^{1}$ National Centre for Infections in Cancer, Peter MacCallum Cancer Institute, Melbourne, Victoria, Australia

${ }^{2}$ National Centre for Antimicrobial Stewardship, Royal Melbourne Hospital, Melbourne, Victoria, Australia

${ }^{3}$ Department of Pharmacy, Peter MacCallum Cancer Institute, Melbourne, Victoria, Australia

${ }^{4}$ Department of General Medicine, Royal Melbourne Hospital, Melbourne, Victoria, Australia

${ }^{5}$ Department of Infectious Diseases, Peter MacCallum Cancer Institute, Melbourne, Victoria, Australia

${ }^{6}$ Center for Health Policy, Melbourne School of Population and Global Health, University of Melbourne, Melbourne, Victoria, Australia

${ }^{7}$ University of Melbourne, Center for Health Policy, Melbourne School of Population and Global Health, Melbourne, Victoria, Australia

${ }^{8}$ Victorian Infectious Diseases Service, Royal Melbourne Hospital, Melbourne, Victoria, Australia

Acknowledgements The authors would like to thank the Sepsis Working Party, clinical nurse leaders (Delia Comodo, Kim Morris, Amy Haffenden, Jessica Willis) 
involved in the process mapping, development and implementation of the sepsis pathway and Ms Sok-Wee Yew for her assistance with data for costings.

Contributors KAT was the clinician lead for the SP since 2012, designed the data collection, contributed to the analysis and was the main author of this paper. SL was the AMS pharmacist and project officer during 2012/2013 and contributed to the study design data collection and authorship. JJ and GMH contributed to the data collection and implementation of the pathway, and review of the manuscript. BT contributed to SP sustainability and to authorship. MT undertook the cost analysis and contributed to authorship. KD supervised cost analysis. GV and AH collected 2014 data. VL contributed to database development and antimicrobial data. CB was the WCMICS project officer who assisted with the hospital implementation at both hospitals. LJW and MAS contributed to study design and authorship.

Funding This study was funded by Western and Central Melbourne Integrated Cancer Centre.

Competing interests None declared.

Patient consent Not required.

Ethics approval Peter MacCallum Cancer Centre Human Research Ethics Committee approved this study (Project No: 13/189 L).

Provenance and peer review Not commissioned; externally peer reviewed.

Data sharing statement Requests for access to data should be addressed to the corresponding author.

Open access This is an open access article distributed in accordance with the Creative Commons Attribution Non Commercial (CC BY-NC 4.0) license, which permits others to distribute, remix, adapt, build upon this work non-commercially, and license their derivative works on different terms, provided the original work is properly cited and the use is non-commercial. See: http://creativecommons.org/ licenses/by-nc/4.0/

(C) Published by the BMJ Publishing Group Limited. For permission to use (where not already granted under a licence) please go to http://www.bmj.com/company/ products-services/rights-and-licensing/

\section{REFERENCES}

1. Kalsekar I, Hsiao CW, Cheng $\mathrm{H}$, et al. Economic burden of cancer among patients with surgical resections of the lung, rectum, liver and uterus: results from a US hospital database claims analysis. Health Econ Rev 2017;7:22.

2. Nazer L, Al-Shaer M, Hawari F. Drug utilization pattern and cost for the treatment of severe sepsis and septic shock in critically ill cancer patients. Int J Clin Pharm 2013;35:1245-50.

3. Kim SP, Shah ND, Karnes RJ, et al. The implications of hospital acquired adverse events on mortality, length of stay and costs for patients undergoing radical cystectomy for bladder cancer. J Urol 2012;187:2011-7.

4. Schilling MB, Parks C, Deeter RG. Costs and outcomes associated with hospitalized cancer patients with neutropenic complications: A retrospective study. Exp Ther Med 2011;2:859-66.

5. Lingaratnam S, Thursky KA, Slavin MA, et al. The disease and economic burden of neutropenic fever in adult patients in Australian cancer treatment centres 2008: analysis of the Victorian Admitted Episodes Dataset. Intern Med J 2011;41:121-9.

6. Kuderer NM, Dale DC, Crawford J, et al. Mortality, morbidity, and cost associated with febrile neutropenia in adult cancer patients. Cancer 2006;106:2258-66.

7. Tam CS, O'Reilly M, Andresen D, et al. Use of empiric antimicrobial therapy in neutropenic fever. Australian Consensus Guidelines 2011 Steering Committee. Intern Med J 2011;41:90-101.

8. Freifeld AG, Bow EJ, Sepkowitz KA, et al. Clinical practice guideline for the use of antimicrobial agents in neutropenic patients with cancer: 2010 update by the infectious diseases society of america. Clin Infect Dis 2011;52:e56-93.

9. Bate J, Gibson F, Johnson E, et al. Neutropenic sepsis: prevention and management of neutropenic sepsis in cancer patients (NICE Clinical Guideline CG151). Arch Dis Child Educ Pract Ed 2013;98:73-5.

10. Thursky KA, Worth LJ. Can mortality of cancer patients with fever and neutropenia be improved? Curr Opin Infect Dis 2015;28:505-13.

11. Bone RC, Balk RA, Cerra FB, et al. Definitions for sepsis and organ failure and guidelines for the use of innovative therapies in sepsis. The ACCP/SCCM Consensus Conference Committee. American College of Chest Physicians/Society of Critical Care Medicine. Chest 1992;101:1644-55.
12. Forde C, Scullin P. Chasing the Golden Hour - Lessons learned from improving initial neutropenic sepsis management. BMJ Qual Improv Rep 2017;6:u204420.w6531.

13. Teles F, Rodrigues WG, Alves M, et al. Impact of a sepsis bundle in wards of a tertiary hospital. J Intensive Care 2017;5:45.

14. Ramsdell TH, Smith AN, Kerkhove E. Compliance with Updated Sepsis Bundles to Meet New Sepsis Core Measure in a Tertiary Care Hospital. Hosp Pharm 2017;52:177-86.

15. Prasad PA, Shea ER, Shiboski S, et al. Relationship Between a Sepsis Intervention Bundle and In-Hospital Mortality Among Hospitalized Patients: A Retrospective Analysis of Real-World Data. Anesth Analg 2017;125:507-13.

16. Leisman DE, Zemmel D'Amore JA, Gribben JL, et al. Early sepsis bundle compliance for non-hypotensive patients with intermediate versus severe hyperlactemia. Am J Emerg Med 2017;35:811-8.

17. Rhodes A, Phillips G, Beale R, et al. The Surviving Sepsis Campaign bundles and outcome: results from the International Multicentre Prevalence Study on Sepsis (the IMPreSS study). Intensive Care Med 2015;41:1620-8.

18. Dellinger RP, Levy MM, Rhodes A, et al. Surviving Sepsis Campaign: international guidelines for management of severe sepsis and septic shock, 2012. Intensive Care Med 2013;39:165-228.

19. Levy MM, Fink MP, Marshall JC, et al. 2001 SCCM/ESICM/ACCP/ ATS/SIS International Sepsis Definitions Conference. Crit Care Med 2003;31:1250-6.

20. James R, Upjohn L, Cotta M, et al. Measuring antimicrobial prescribing quality in Australian hospitals: development and evaluation of a national antimicrobial prescribing survey tool. $J$ Antimicrob Chemother 2015;70:1912-8.

21 Curry L, Nunez-Smith M. Mixed methods in Health Sciences A practical Primer. Los Angeles: Sage, 2015.

22. Development ACfC. The International Statistical Classification of Diseases and Related Health Problems, tenth revision, Australian modification (ICD-10-AM/ACHI/ACS). Independent Hospital Pricing Authority: Darlinghurst NSW, 2017.

23. Statistics ABo. 6401.0 - Consumer Price Index 2017. http://www abs.gov.au/ausstats/abs@.nsf/mf/6401.0

24. Commission NCE. Sepsis Kills NSW. http://www.cec.health.nsw.gov. au/patient-safety-programs/adult-patient-safety/sepsis-kills

25. Porteous JM, Stewart-Wynne EG, Connolly M, et al. iSoBAR--a concept and handover checklist: the National Clinical Handover Initiative. Med J Aust 2009;190(11 Suppl):S152-6.

26. Talmor D, Greenberg D, Howell MD, et al. The costs and costeffectiveness of an integrated sepsis treatment protocol. Crit Care Med 2008;36:1168-74

27. Jones AE, Troyer JL, Kline JA. Cost-effectiveness of an emergency department-based early sepsis resuscitation protocol. Crit Care Med 2011;39:1306-12.

28. Suarez D, Ferrer R, Artigas A, et al. Cost-effectiveness of the Surviving Sepsis Campaign protocol for severe sepsis: a prospective nation-wide study in Spain. Intensive Care Med 2011;37:444-52.

29. Assuncao MS, Teich V, Shiramizo SC, et al. The cost-effectiveness ratio of a managed protocol for severe sepsis. J Crit Care 2014;29:692.e1-6.

30. Tarrant C, O'Donnell B, Martin G, et al. A complex endeavour: an ethnographic study of the implementation of the Sepsis Six clinical care bundle. Implement Sci 2016;11:149.

31. Roberts N, Hooper G, Lorencatto F, et al. Barriers and facilitators towards implementing the Sepsis Six care bundle (BLISS-1): a mixed methods investigation using the theoretical domains framework. Scand J Trauma Resusc Emerg Med 2017;25:96.

32. Hiong A, Thursky KA, Teh BW, et al. Sepsis following cancer surgery: the need for early recognition and standardised clinical care. Expert Rev Anti Infect Ther 2016;14:425-33.

33. Bruce HR, Maiden J, Fedullo PF, et al. Impact of nurse-initiated ED sepsis protocol on compliance with sepsis bundles, time to initial antibiotic administration, and in-hospital mortality. J Emerg Nurs 2015;41:130-7.

34. Jones SL, Ashton CM, Kiehne L, et al. Reductions in Sepsis Mortality and Costs After Design and Implementation of a Nurse-Based Early Recognition and Response Program. Jt Comm J Qual Patient Saf 2015;41:483-91.

35. McGaughey J, O'Halloran P, Porter S, et al. Early warning systems and rapid response to the deteriorating patient in hospital: A realist evaluation. J Adv Nurs 2017;73:3119-32.

36. Braithwaite J, Westbrook J, Coiera E, et al. A systems science perspective on the capacity for change in public hospitals. Isr J Health Policy Res 2017;6:16.

37. Serafim R, Gomes JA, Salluh J, et al. A Comparison of the QuickSOFA and Systemic Inflammatory Response Syndrome Criteria for 
the Diagnosis of Sepsis and Prediction of Mortality: A Systematic Review and Meta-Analysis. Chest 2018;153:646-55.

38. Williams JM, Greenslade JH, McKenzie JV, et al. Systemic Inflammatory Response Syndrome, Quick Sequential Organ Function Assessment, and Organ Dysfunction: Insights From a Prospective Database of ED Patients With Infection. Chest 2017;151:586-96.

39. Churpek MM, Snyder A, Han X, et al. Quick Sepsis-related Organ Failure Assessment, Systemic Inflammatory Response Syndrome, and Early Warning Scores for Detecting Clinical Deterioration in Infected Patients outside the Intensive Care Unit. Am J Respir Crit Care Med 2017;195:906-11.

40. Manaktala S, Claypool SR. Evaluating the impact of a computerized surveillance algorithm and decision support system on sepsis mortality. J Am Med Inform Assoc 2017;24.

41. Makam AN, Nguyen OK, Auerbach AD. Diagnostic accuracy and effectiveness of automated electronic sepsis alert systems: A systematic review. J Hosp Med 2015;10:396-402.

42. Berger T, Birnbaum A, Bijur P, et al. A Computerized Alert Screening for Severe Sepsis in Emergency Department Patients Increases
Lactate Testing but does not Improve Inpatient Mortality. App/ Clin Inform 2010;1:394-407.

43. Jolley RJ, Quan H, Jetté N, et al. Validation and optimisation of an ICD-10-coded case definition for sepsis using administrative health data. BMJ Open 2015;5:e009487.

44. Chin YT, Scattergood N, Thornber M, et al. Accurate coding in sepsis: clinical significance and financial implications. J Hosp Infect 2016;94:99-102.

45. Damiani E, Donati A, Serafini G, et al. Effect of performance improvement programs on compliance with sepsis bundles and mortality: a systematic review and meta-analysis of observational studies. PLoS One 2015;10:e0125827.

46. Thomas BS, Jafarzadeh SR, Warren DK, et al. Temporal trends in the systemic inflammatory response syndrome, sepsis, and medical coding of sepsis. BMC Anesthesiol 2015;15:169.

47. Burston J, Adhikari S, Hayen A, et al. A Role for Antimicrobial Stewardship in Clinical Sepsis Pathways: a Prospective Interventional Study. Infect Control Hosp Epidemiol 2017;38:1032-8 